21

\title{
Оптимизация параметров и характеристик широкополосных терагерцовых поглотителей на основе $2 d$-решеток графеновых лент на многослойных подложках
}

\author{
(C) А.М. Лерер ${ }^{1}$, И.Н. Иванова ${ }^{1}$, Г.С. Макеева ${ }^{2}$, В.В. Черепанов ${ }^{1}$ \\ ${ }^{1}$ Южный федеральный университет, физический фракультет, \\ 344090 Ростов-на-Дону, Россия \\ ${ }^{2}$ Пензенский государственный университет, \\ 440026 Пенза, Россия \\ e-mail: lerer@sfedu.ru, radiotech@pnzgu.ru
}

Поступила в редакцию 10.08.2020 г.

В окончательной редакции 05.11.2020 г.

Принята к публикации 19.11.2020 г.

Теоретически исследованы многослойные двумерно-периодические решетки графеновых микролент, которые при резонансе поверхностного плазмон-поляритона поглощают почти 100\% падающей на нее энергии. Для повышения широкополосности поглотителей предлагается в элементарной ячейке $2 d$-решетки располагать 2-3 параллельные графеновые ленты с близкими длинами.

Ключевые слова: многослойные поглотители, графеновые ленты, ТГц, широкополосность, плазмонный резонанс.

DOI: $10.21883 /$ OS.2021.03.50661.217-20

\section{Введение}

Исследования в области терагерцовых (THz) технологий получают все большее внимание также из-за их разнообразных приложений в беспроводной связи и системах передачи информации, сфере безопасности, астрономии, спектроскопии $[1,2]$. Интенсивные усилия были сделаны для разработки и изготовления новых многофункциональных $\mathrm{THz}$ устройств. Из-за важной роли в визуализации, зондировании и обнаружении поглотители являются одними из самых востребованных компонентов в $\mathrm{THz}$ диапазоне [1,3].

Большой интерес вызвали поглотители на основе резонанса в метаматериалах [4]. Однако в этих структурах используются благородные металлы, которые характеризуются тем, что их электрические и электромагнитные свойства трудно перестраиваются, особенно когда требуются тонкие пленки. Более того, большинство этих структур непрозрачны в видимом диапазоне, что сужает область их применения.

Подобно благородным металлам графен перспективен для плазмонных устройств [2,5]. Поверхностные плазмон-поляритоны (ППП) в графене предлагают сильное ограничение поля и относительно большее расстояние распространения. Кроме того, плазмонные свойства графена могут быть управляемы электрически или химически. Эти особенности графена делают его многообещающим для многих приложений, таких как оптические модуляторы, датчики и поглотители [6-8].

В последнее время быстро растущий интерес к реализации привлекают ультракомпактные $\mathrm{THz}$ поглотители на основе графена с перестраиваемыми абсорбционными свойствами, регулируемыми приложенным к затвору напряжением или химическим легированием [1].

Численные исследования показывают, что, используя решетки графеновых лент, связанные с графеновыми листами, можно добиться поглощения, близкого к единице в широкой полосе $\mathrm{THz}$ частот, путем изменения электрических свойств графена с помощью химического потенциала [1,9-12].

Благодаря огромному потенциалу в практической инженерии значительное внимание уделяется $\mathrm{THz}$ поглотителям на основе графена для широкополосных применений, с такими характеристиками как двухполосные [13], многополосные [2,14,15], сверхширокополосные $[16,17]$ и управляемые поглотители $[2,3,18,19]$. Однако некоторые ключевые вопросы до сих пор не решены. Необходимы системный анализ и проектирование сверхширокополосных поглотителей на основе многослойных графеновых решеток с элементами произвольной конфигурации из-за их способности поддерживать множественные плазмонные резонансы [2], в том числе с учетом в методах проектирования не только нормального падения волны, но и под углом для разработки поглотителей с лучшей поляризационной и угловой нечувствительностью характеристик $[1,10,16,18]$.

Настоящая статья - развитие наших работ, посвященных графеновым поглотителям $[11,12]$. Эти поглотители на основе двумерно-периодических решеток графеновых лент конечной длины, расположенных на верхнем диэлектрическом слое и нескольких чередующихся слоях графена и диэлектрика. Отмечено, что максимальное поглощение наблюдается вблизи частот резонансов ППП на графеновых лентах. В данной работе для повышения 
широкополосности устройства предлагается в одной элементарной ячейке решетки располагать 2-3 параллельные графеновые ленты с близкими длинами. При этом несколько близких по частоте резонансов поглощения сливаются в один с относительной шириной около $10 \%$ по уровню поглощения 90\%.

В графеновых, как и вообще в оптических плазмонных структурах, не работает принцип масштабируемости - свойства графена зависят от частоты. Поэтому для каждого диапазона частот приходится подбирать размеры элементов решетки. На резонансную частоту прежде всего влияют периоды $2 d$-решетки, длина лент и параметры графена. На локализацию поля вблизи графеновых лент, а значит, и на величину поглощения влияют также толщины диэлектрических слоев. Эти слои можно назвать согласующими. Выбор параметров этих слоев - одна из задач синтеза поглотителей.

В настоящей работе мы поставили задачи:

$\checkmark$ улучшить полученные ранее характеристики $\mathrm{THz}$ поглотителей, оптимизируя толщины согласующих диэлектрических слоев;

• изучить влияние параметров графена и размеров решетки на характеристики поглотителя;

$\checkmark$ исследовать возможности полного поглощения при частотах выше $1 \mathrm{THz}$;

$\checkmark$ исследовать зависимость поглощения от угла падения волны.

\section{Вычислительные методы}

Для теоретического исследования $1 d$ - и $2 d$-графеновых решеток используются два метода и комплекс программ для электродинамического моделирования плазмонных дифракционных решеток [20-23]. Краткое изложение этих методов приведено в приложении.

Первый - метод объемного интегро-дифференциального уравнения (ОИДУ) [20]. Для интеграции графеновых слоев в разработанный комплекс программ слой графена, на котором выполняются импедансные граничные условия Кубо, заменяется тонким слоем диэлектрика, диэлектрическая проницаемость которого определяется следующим образом:

$$
\varepsilon=1-i \frac{Z_{0} \sigma}{k t}
$$

где $\sigma$ - поверхностная проводимость графена, $k, Z_{0}-$ волновое число и волновое сопротивление в вакууме. В (1) фигурирует толщина слоя $t$. Она может быть любой. Единственное условие $-t \ll \lambda_{0}\left(\lambda_{0}-\right.$ длина волны в вакууме).

Второй - метод, использующий приближенные граничные условия (ПГУ) для тонких плазмонных структур [21]. Основное отличие - использование формулы Кубо для проводимости графенового слоя.

Расчет графеновых поглотителей двумя независимыми методами позволяет, во-первых, проверить достоверность полученных теоретических результатов, во-вторых, модифицировать большой комплекс программ электродинамического моделирования диэлектрических и плазмонных структур для расчета графеновых структур.

\section{Результаты моделирования}

Начнем с исследованных ранее в [12] ТНz поглотителей (рис. 1). Они имеют девять чередующих слоев диэлектрика и графена и 3 графеновые полоски (поз. 1) одинаковой ширины $w$ и разных длин $l_{1}, l_{2}, l_{3}$ в одной элементарной ячейке. Показатель преломления диэлектрических слоев 1.77, полубесконечной подложки 1.45 . Параметры графеновых слоев такие же, как параметры полосок. В отличие от [12] а) исследовано влияние химического потенциала $\mu_{c}$ и ширины полосок на характеристики поглотителя; б) для увеличения поглощения проведена оптимизация толщин согласующих диэлектрических слоев $h_{d}$; в) исследованы поглотители в более широком интервале размеров и диапазоне $\mathrm{THz}$ частот.

При $s$-поляризации волны (поляризация падающей волны вдоль широкой стороны лент) наблюдается продольный плазмонный резонанс, при котором максималь-

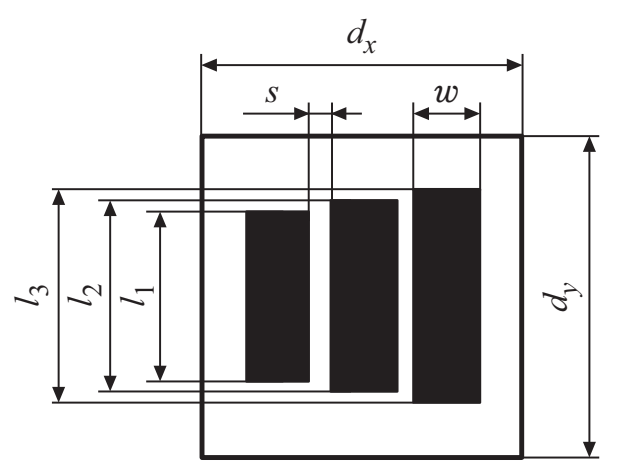

$a$

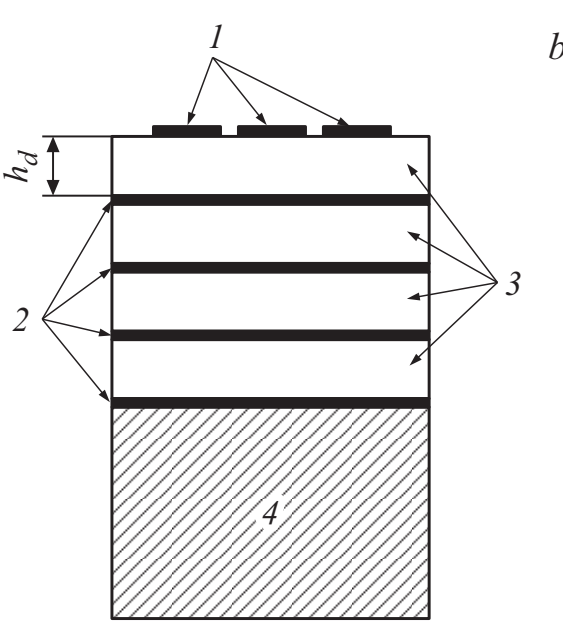

Рис. 1. Элементарная ячейка многослойной двумернопериодической решетки с периодами $d_{x}, d_{y}$. (a) Вид сверху, (b) вид сбоку. 1 - графеновые микроленты с шириной $w$ и длинами $l_{1}, l_{2}, l_{3}$, расположенные с зазором $s ; 2-$ монослои графена, 3 - разделительные диэлектрические слои толщиной $h_{d}, 4-$ полубесконечная подложка. 


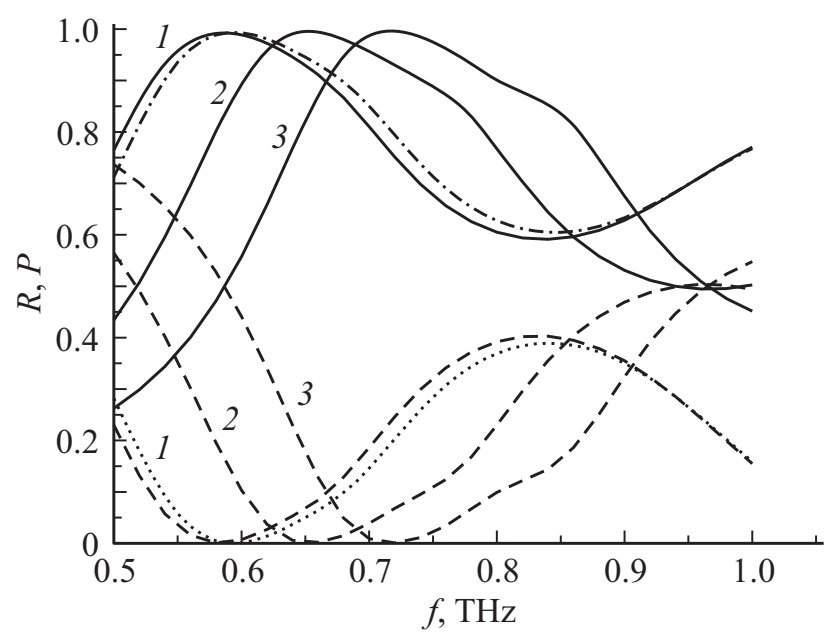

Рис. 2. Частотные характеристики $\mathrm{THz}$ поглотителя на основе многослойной решетки графеновых лент при изменении химического потенциала $\mu_{c}$ графена: сплошные и штрихпунктирная кривые - коэффициент поглощения $P_{s}$, штриховые и пунктирная - коэффициент отражения по мощности $R_{s} ; s-$ поляризация, $d_{x}=d_{y}=70 \mu \mathrm{m}, w=15 \mu \mathrm{m}, l_{1}=50, l_{2}=45$ и $l_{3}=40 \mu \mathrm{m}$. Расстояние между лентами $s=5 \mu \mathrm{m}$. Кривые 1 : $\mu_{c}=0.25 \mathrm{eV}, h_{d}=25 \mu \mathrm{m}$; кривые $2: \mu_{c}=0.35 \mathrm{eV}, h_{d}=23 \mu \mathrm{m}$; кривые 3: $\mu_{c}=0.4 \mathrm{eV}, h_{d}=20 \mu \mathrm{m}$. Сплошные и штриховые кривые - метод ОИДУ, пунктирная и штрихпунктирная метод ПГУ.

на плотность тока на графеновых лентах и поэтому максимально и поглощение (рис. 2). Резонансная частота зависит от периодов, размеров элементов решетки и параметров графена. Из рисунка видна значительная перестройка по частоте максимума поглощения при изменении химического потенциала $\mu_{c}$. Ширина резонансной кривой на уровне 0.9 около $20 \%$.

При изменении ширины лент и неизменных других размерах резонансная частота изменяется, уменьшается поглощение. Для достижения полного поглощения при плазмонном резонансе нужно изменить толщины согласующих слоев $h_{d}$ (рис. 3). В результате резонансные частоты по сравнению с поглотителем рис. 2 не изменяются, но при малых значениях химического потенциала $\mu_{c}$ появляется второй резонанс поглощения.

У прямоугольной ленты продольный при $s$-поляризации (рис. 2, 3) и поперечный при р-поляризации (рис. 4) резонансы разнесены по частоте. Поэтому при частоте продольного резонанса коэффициент отражения по мощности $R_{s}$ волны с $s$-поляризацией близок к нулю (рис. 2, 3), а у ортогональной $p$-поляризации $R_{p}$ больше 0.6 (рис. 4). Таким образом, графеновый поглотитель является также $\mathrm{THz}$ поляризатором с развязкой $N=10 \lg \left(R_{p} / R_{s}\right)$ между волнами с $s$ - и $p$-поляризацией по коэффициенту отражения более $20 \mathrm{~dB}$. Указанные свойства сохраняются в достаточно широком интервале углов падения $\pm 30^{\circ}$ (рис. 5). Из результатов следует, что характеристики $\mathrm{THz}$ поглотителя и поляризатора обладают достаточной поляризационной (рис. 5,a) и угловой (рис. 5, $b$ ) нечувствительностью.

Расчетные зависимости поверхностной проводимости $\sigma_{s}$ графена от частоты в широком интервале частот от $1 \mathrm{GHz}$ до $100 \mathrm{THz}$, который включает СВЧ, ТНz и ИК диапазоны, показаны на рис. 6. Видно, что в интервале частот $0.01-0.1 \mathrm{THz}$ действительная часть $\sigma_{s}^{\prime}$ поверхностной проводимости графена является практически постоянной величиной, а в интервале частот $0.5-10 \mathrm{THz}$

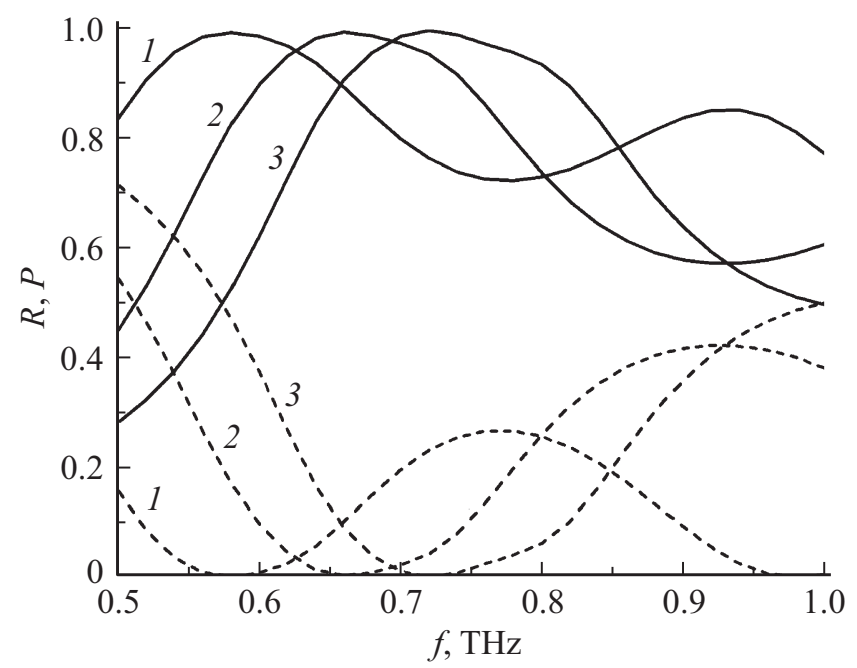

Рис. 3. Частотные характеристики ТНz поглотителя при изменении ширины графеновых лент для разных значений химического потенциала $\mu_{c}$ : сплошные кривые $-P_{s}$, штриховые $R_{s} ; s-$ поляризация, $d_{x}=d_{y}=70 \mu \mathrm{m}, w=10 \mu \mathrm{m}, l_{1}=50$, $l_{2}=45$ и $l_{3}=40 \mu \mathrm{m}$. Кривые $1-\mu_{c}=0.25 \mathrm{eV}, h_{d}=30 \mu \mathrm{m}$; кривые $2-\mu_{c}=0.35 \mathrm{eV}, h_{d}=25 \mu \mathrm{m}$; кривые $3-\mu_{c}=0.4 \mathrm{eV}$, $h_{d}=23 \mu \mathrm{m}$.

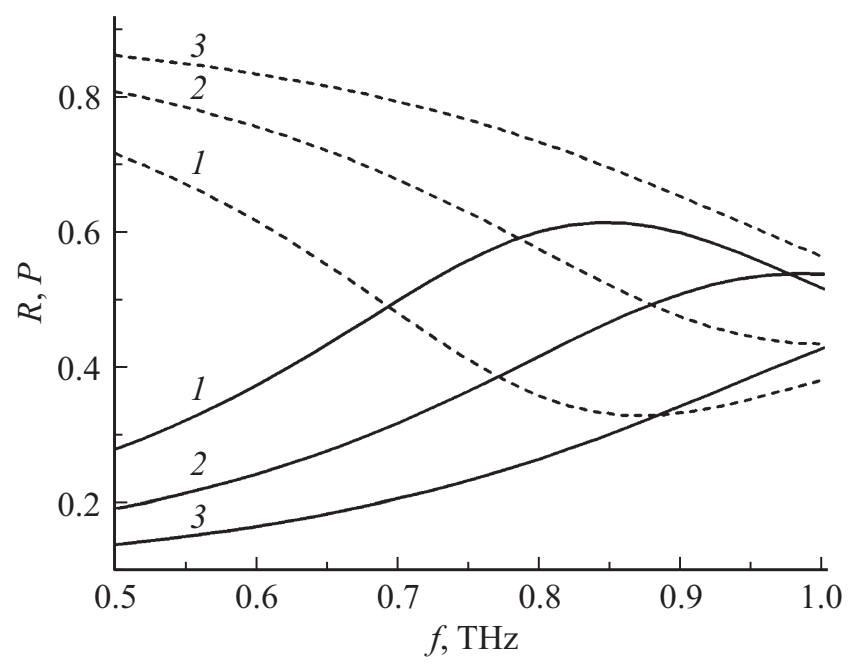

Рис. 4. Частотные характеристики $\mathrm{THz}$ поглотителя для волны $p$-поляризации при разных значениях химического потенциала $\mu_{c}$ : сплошные кривые $-P_{p}$, штриховые $-R_{p}$. Кривые $1-\mu_{c}=0.25 \mathrm{eV}, h_{d}=25 \mu \mathrm{m}$; кривые $2-\mu_{c}=0.35 \mathrm{eV}$, $h_{d}=23 \mu \mathrm{m}$; кривые $3-\mu_{c}=0.4 \mathrm{eV}, h_{d}=20 \mu \mathrm{m}$. Параметры и размеры поглотителя см. на рис. 2. 

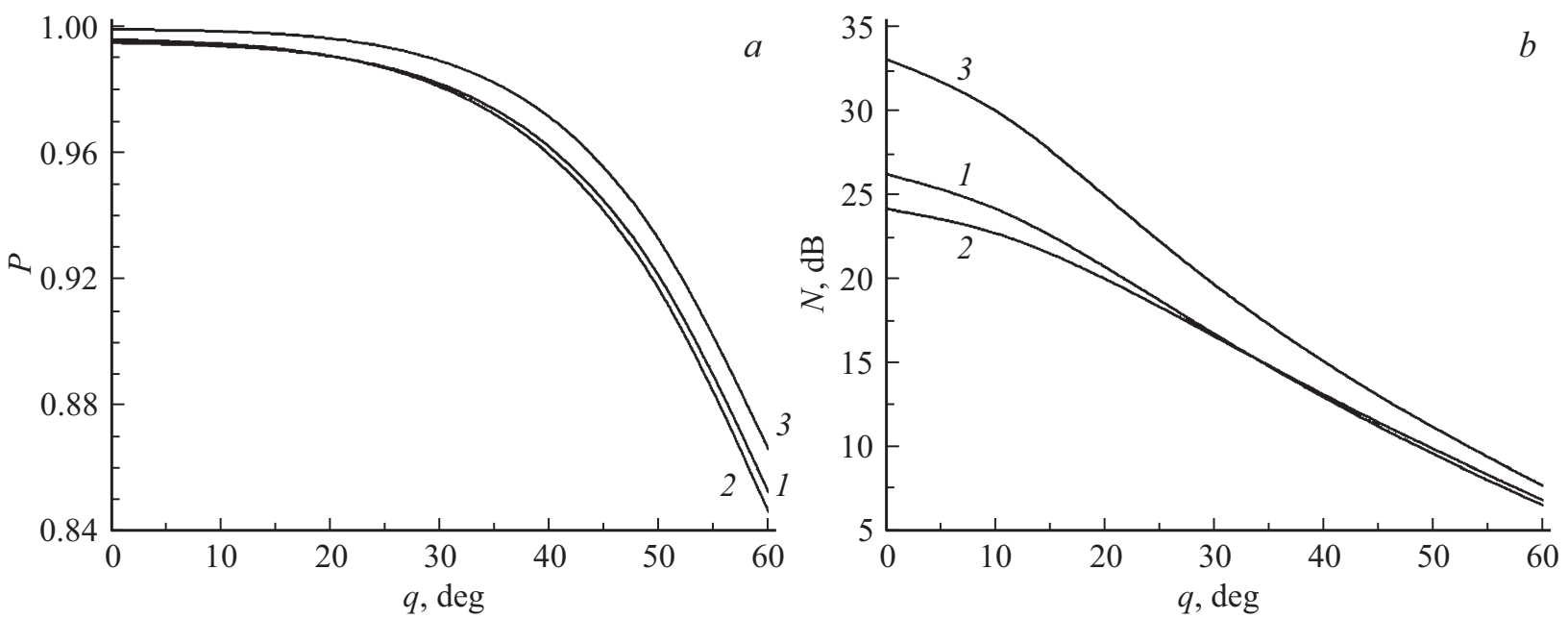

Рис. 5. Поляризационная и угловая чувствительности характеристик $\mathrm{THz}$ поглотителя и поляризатора: угловые зависимости коэффициента поглощения $P_{s}, s$-поляризация $(a)$ и развязки $N$ между поляризациями $(b)$ при разных значениях химического потенциала $\mu_{c}$. Кривые $1-\mu_{c}=0.25 \mathrm{eV}, h_{d}=30 \mu \mathrm{m}$; кривые $2-\mu_{c}=0.35 \mathrm{eV}, h_{d}=25 \mu \mathrm{m}$; кривые $3-\mu_{c}=0.4 \mathrm{eV}, h_{d}=23 \mu \mathrm{m}$. Размеры и параметры устройства как на рис. 2.

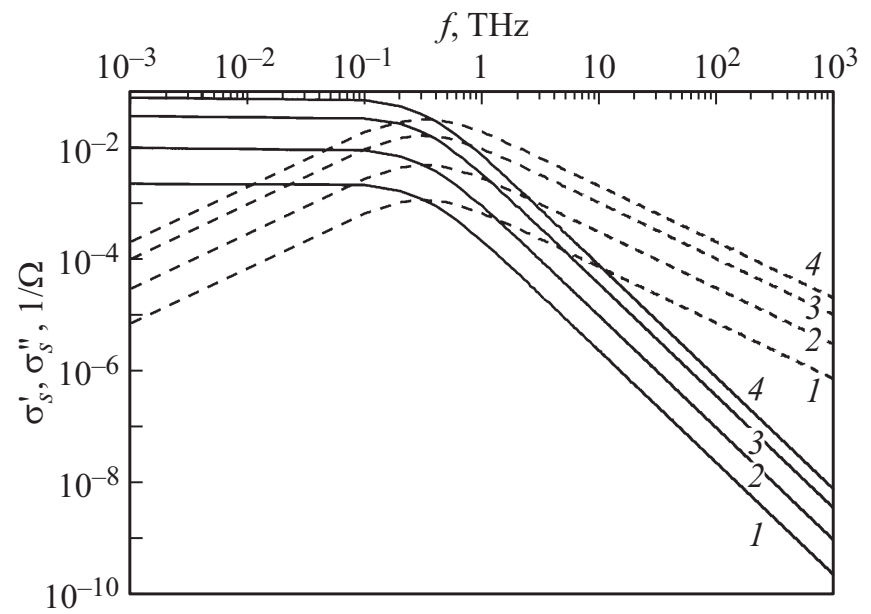

Рис. 6. Частотные зависимости действительной $\sigma_{s}^{\prime}$ и мнимой $\sigma_{s}^{\prime \prime}$ частей поверхностной проводимости графена в СВЧ, $\mathrm{THz}$ и ИК диапазонах при различных значениях химического потенциала: $1-\mu_{c}=0 \mathrm{eV}, 2-\mu_{c}=0.15 \mathrm{eV}, 3-\mu_{c}=0.5 \mathrm{eV}$, $4-\mu_{c}=1 \mathrm{eV}$. Сплошные линии - $\sigma_{s}^{\prime}$, штриховые $-\sigma_{s}^{\prime \prime}$.

существенно уменьшаются с ростом частоты. При изменении значения химического потенциала $\mu_{c}$ от 0 до $1 \mathrm{eV}$ поверхностная проводимость графена $\sigma_{s}^{\prime}$ в $\mathrm{THz}$ диапазоне увеличивается почти в 20 раз.

Вследствие частотной зависимости поверхностной проводимости $\sigma_{s}^{\prime}$ графена изменение размеров элементов решетки в два раза приводит, естественно, к сдвигу частоты резонанса, но это изменение не пропорционально изменению размеров (рис. 7-9).

Как следует из результатов рис. 7-9, по мере увеличения химического потенциала (уровня Ферми) и уменьшения периода решетки графеновых лент частота плазмонного резонанса и, следовательно, максимум поглощения проявляют сдвиг в сторону более высоких $\mathrm{THz}$ частот. Фактор качества (добротность) резонанса и, следовательно, эффективность поглощения также повышаются с уменьшением химического потенциала графена (уровня Ферми), однако снижаются из-за уменьшения периода решетки, поэтому для увеличения эффективности поглощения при плазмонном резонансе нужно изменить толщины согласующих слоев $h_{d}$.

Увеличение периода решетки приводит к уменьшению частоты плазмонного резонанса (рис. 7). Умень-

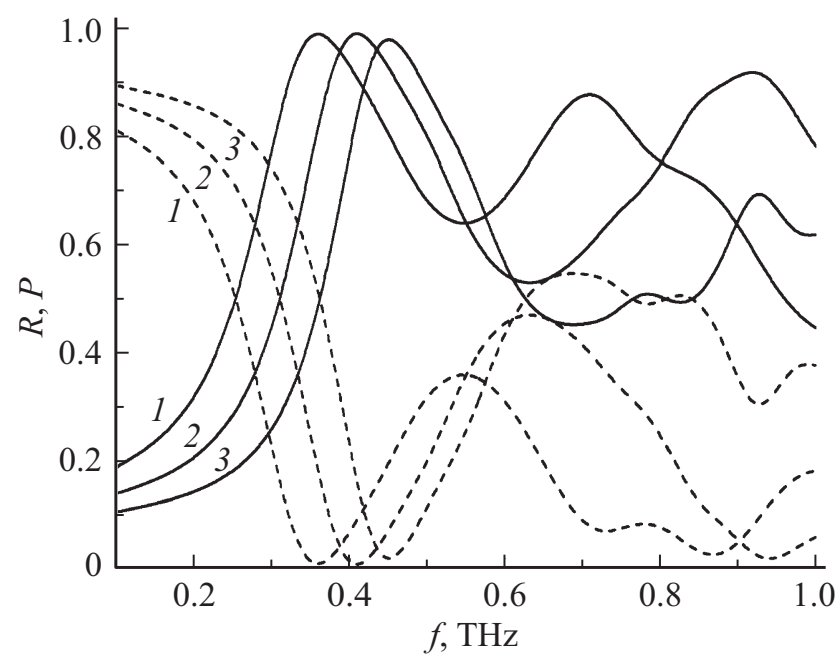

Рис. 7. Частотные зависимости коэффициента поглощения $P_{s}$ (сплошные кривые) и коэффициента отражения по мощности $R_{s}$ (штриховые) при увеличении геометрических размеров элементов решетки для разных значений химического потенциала $\mu_{c}$ : $s$-поляризация, $d_{x}=d_{y}=140 \mu \mathrm{m}, w=30 \mu \mathrm{m}, l_{1}=100$, $l_{2}=90$ и $l_{3}=80 \mu \mathrm{m}, s=10 \mu \mathrm{m}$. Кривые $1-\mu_{c}=0.25 \mathrm{eV}$, $h_{d}=50 \mu \mathrm{m}$; кривые $2-\mu_{c}=0.35 \mathrm{eV}, h_{d}=40 \mu \mathrm{m}$; кривые $3-$ $\mu_{c}=0.4 \mathrm{eV}, h_{d}=30 \mu \mathrm{m}$. 


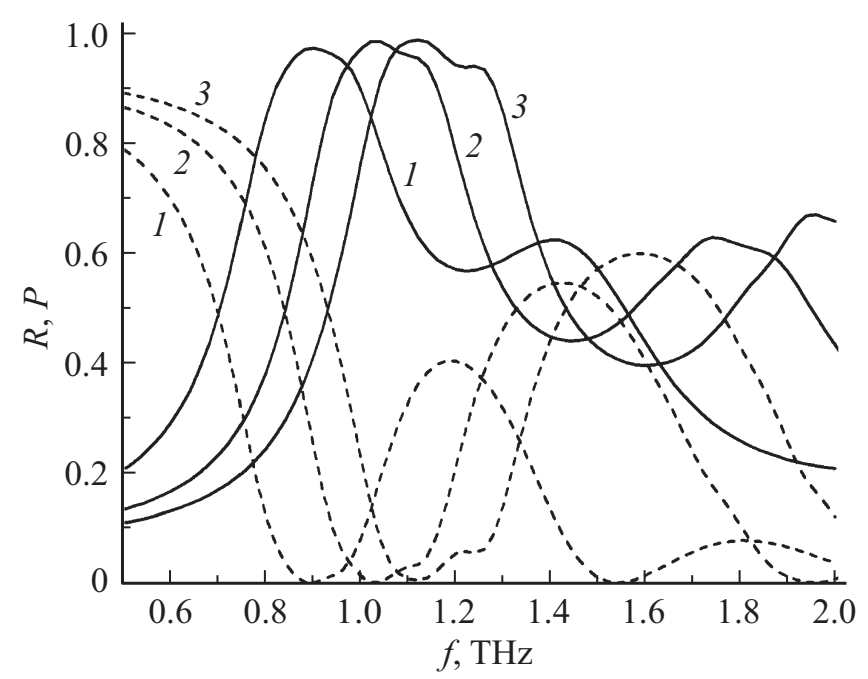

Рис. 8. Частотные зависимости коэффициента поглощения $P_{s}$ (сплошные кривые) и коэффициента отражения по мощности $R_{s}$ (штриховые) при уменьшении геометрических размеров элементов решетки для разных значений химического потенциала $\mu_{c}: s$-поляризация $d_{x}=d_{y}=35 \mu \mathrm{m}, h_{d}=15 \mu \mathrm{m}$ (кривые 1$), 12.5 \mu \mathrm{m}(2,3) . w=7.5 \mu \mathrm{m} ; l_{1}=25 \mu \mathrm{m}, l_{2}=22.5 \mu \mathrm{m}$, $l_{3}=20 \mu \mathrm{m} ; s=2.5 \mu \mathrm{m}$

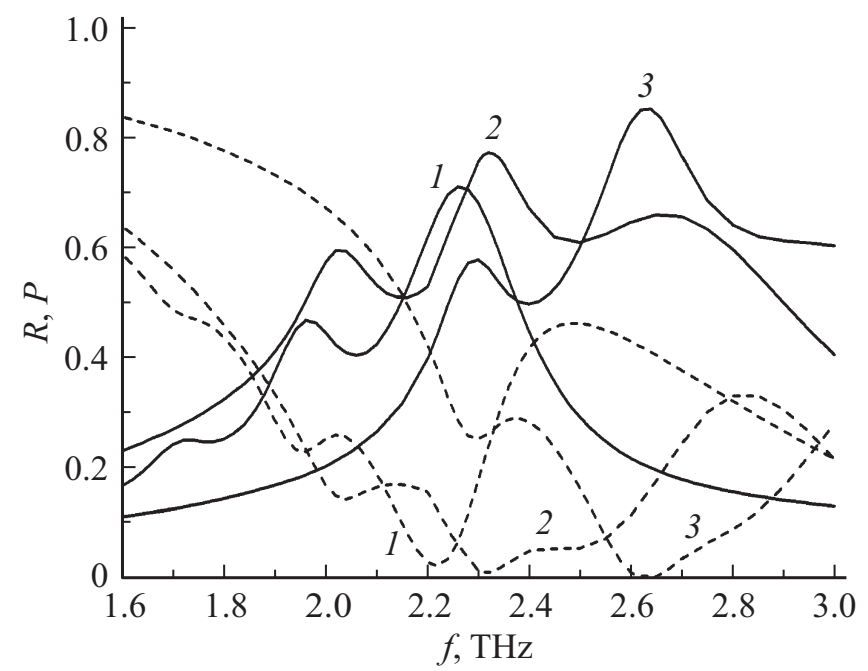

Pис. 9. Частотные зависимости коэффициента поглощения $P_{s}$ (сплошные кривые) и коэффициента отражения по мощности $R_{s}$ (штриховые) при уменьшении геометрических размеров элементов решетки для разных значений химического потенциала $\mu_{c}: s$-поляризация, $d_{x}=d_{y}=7 \mu \mathrm{m}, h_{d}=13 \mu \mathrm{m}$ (кривые 1$), 5 \mu \mathrm{m}(2,3) ; w=1 \mu \mathrm{m} ; l_{1}=5 \mu \mathrm{m}, l_{2}=4.5 \mu \mathrm{m}$, $l_{3}=4 \mu \mathrm{m} ; s=0.5 \mu \mathrm{m}$.

шение периода (рис. 8, 9) увеличивает резонансную частоту. Как видно из рис. 8, 9, при частотах выше $1 \mathrm{THz}$ не удалось получить $100 \%$ поглощения. Это обусловлено резким уменьшением действительной части $\sigma_{s}^{\prime}$ поверхностной проводимости и, следовательно, потерь в графене на частотах больше $1 \mathrm{THz}$ (рис. 6).

\section{Заключение}

В поглотителях на основе двумерно-периодических решеток графеновых лент на многослойных графендиэлектрических подложках плазмонные резонансы обеспечивают эффективный способ усилить взаимодействие $\mathrm{THz}$ излучения с графеном. Эффективность этого взаимодействия сильно зависит от химического потенциала графена и от параметров окружающей диэлектрической среды. Влияние окружающих графен диэлектрических слоев, их размеров и параметров, конфигурации структуры на эффективность поглощения при плазмонном резонансе дает множество дизайнерских возможностей, которые можно реализовать с помощью доступных технологий нанофотоники.

Графеновые плазмонные наноструктуры разрабатываются, чтобы решить ключевую, остающуюся неразрешенной проблему современной оптоэлектроники это ограниченная практическая доступность высокоэффективных THz устройств. Если соответствующие уровни нанотехнологий будут достигнуты, графеновые ТHz поглотители перспективны по нескольким причинам, общим для других графеновых технологий: они могут изготавливаться с помощью относительно простых и недорогих технологических процессов (например, не требующих какого-либо сложного гетероэпитаксиального роста), напрямую совместимы с широким спектром подложек (включая микроэлектронные схемы на основе $\mathrm{Si}$ ), способны обеспечить экстремальные размеры миниатюризации и внести свой вклад в появление новых $\mathrm{THz}$ устройств на чипах для высокоскоростной обработки информации.

Работа выполнена при финансовой поддержке Министерства науки и высшего образования Российской Федерации (Государственное задание в области научной деятельности, Южный федеральный университет, 2020 г.)

\section{Приложение. Методы решения краевой задачи дифракции электромагнитной волны на двумерно-периодических решетках}

\section{1. Метод объемных интегро-дифференциальных уравнений относительно напряженности электрического поля внутри диэлектрического тела (Оиду)}

ОИДУ имеют ряд преимуществ: они более простые, чем поверхностные ИДУ (ПИДУ), неоднородность и нелинейность диэлектрика не усложняет существенно решение, в результате решения непосредственно находится электрическое поле в диэлектрике. Однако размерность ОИДУ выше, чем размерность ПИДУ. Поэтому 
при их численной реализации необходимо выбирать оптимальный базис для каждого тела.

ОИДУ бисингулярное. В наших работах [20,23] изложен эффективный метод расчета оптических наноструктур, основанный на численно-аналитическом решении ОИДУ. В основе метода использование интегрального представления функции Грина. Особенность ядра ОИДУ проявляется в медленной сходимости интегралов или рядов в матричных элементах полученной методом Галеркина системы линейных алгебраических уравнений (СЛАУ). Улучшить сходимость интегралов проще, чем регуляризовать ОИДУ в пространственном представлении.

Решение ОИДУ можно разбить на три этапа:

а) для задач дифракции решение задачи о прохождении и отражении волны от многослойной структуры без неоднородностей;

b) решение методом Галеркина с базисом, оптимальным для исследуемой структуры;

с) улучшение сходимости рядов или интегралов, через которые выражаются матричные элементы полученных систем линейных алгебраических уравнений.

Рассмотрим вначале ОИДУ для диэлектрического тела в однородной среде с диэлектрической проницаемостью, равной единице. Полагаем, что магнитная проницаемость тела равна единице

$$
\mathbf{E}(\mathbf{r})=\mathbf{E}^{\mathrm{BH}}(\mathbf{r})+\left(\operatorname{grad} \operatorname{div}+k^{2}\right) \int_{V}(\varepsilon-1) \mathbf{E}\left(\mathbf{r}^{\prime}\right) G\left(\mathbf{r}, \mathbf{r}^{\prime}\right) d v^{\prime},
$$

где $\varepsilon(\mathbf{r})$ - диэлектрическая проницаемость тела, $V$ объем тела, $k$ - волновое число,

$$
G\left(\mathbf{r}, \mathbf{r}^{\prime}\right)=\frac{1}{4 \pi} \frac{\exp \left(-i k r\left|\mathbf{r}-\mathbf{r}^{\prime}\right|\right)}{\left|\mathbf{r}-\mathbf{r}^{\prime}\right|}
$$

- функция Грина.

ОИДУ для диэлектрического тела $V$ на или внутри многослойной подложке более сложное. В качестве примера ОИДУ для двухмерно-периодической (периоды $\left.d_{x}, d_{y}\right)$ гексагональной решетки из диэлектрических неоднородностей

$$
\begin{gathered}
E(x, y, z)=\mathbf{E}^{\mathrm{BH}}(x, y, z)+\frac{1}{d_{x} d_{y}} \int_{V} d v^{\prime} \tau \\
\times \sum_{p=-\infty}^{\infty} \sum_{q=-\infty}^{\infty} \mathbf{G}_{p q}\left(z, z^{\prime}\right) \mathbf{E}\left(x^{\prime}, y^{\prime}, z^{\prime}\right) \exp \left[\left(\alpha_{p} \bar{x}+\beta_{q} \bar{y}\right)\right] \\
x, y, z \in V,
\end{gathered}
$$

где $\bar{x}=x-x^{\prime}, \bar{y}=y-y^{\prime}, \alpha_{p}=\frac{2 p \pi}{d_{x}}+k_{x}, \beta_{p, q}=\frac{2 p \pi}{d_{y}}+k_{y}$, $k_{x, y}$ - проекции волнового вектора, $V$ - объем неоднородностей, образующих решетку. В одной элементарной ячейке может быть несколько неоднородностей. $\tau=\left(\varepsilon-\varepsilon^{\mathrm{BH}}\right) / \varepsilon^{\mathrm{BH}}, \varepsilon, \varepsilon^{\mathrm{BH}}-$ диэлектрические проницаемости тела и слоя с координатой $z^{\prime}$. Если слои или неоднородности - графеновые, то они заменяются диэлектрическими с эквивалентными $\varepsilon$ и толщиной, полученными из формулы Кубо. Если слои или неоднородности плазмонные (например, $\mathrm{Au}, \mathrm{Ag}$ и т.д.), то значения $\varepsilon(\lambda)$ - из опубликованных баз данных. $\mathbf{E}^{\text {вн }}$ - напряженность внешнего электрического поля, под которым подразумеваем поле, полученное в результате решения задачи об отражении волны плоской от исследуемой структуры, в которой убраны неоднородности.

Элементы дифференциального тензорного ядра $\mathbf{G}_{p q}\left(z, z^{\prime}\right)$ получены в [20] для произвольного числа слоев. Практические расчеты для решеток с 123 слоями.

ИДУ (П1) решаем методом Галеркина. Решение ищем в виде

$$
E_{r}(x, y, z)=\sum_{l=0_{x}}^{N_{x}} \sum_{m=0}^{N_{y}} \sum_{n=1}^{N_{s}} X_{l m n}^{r} V_{l m n}(x, y, z),
$$

где $X_{l m n}^{r}-$ неизвестные коэффициенты, $V_{l m n}(x, y, z)-$ базисные функции (БФ),

$$
V_{l m n}(x, y, z)=R_{l m}(x, y, z) Z_{n}(z),
$$

$R_{l m}(x, y, z)$ - БФ, зависящие от поперечных координат $(x, y)$. Если поперечное сечение неоднородности нерегулярное, т.е. зависящее от продольной координаты, перпендикулярной поверхности подложки $z$, то $R_{l m}(x, y, z)$ зависит также и от $z$.

Для неоднородностей с прямоугольным поперечным сечением $2 l_{x}(z) \times 2 l_{x}(z)$

$$
R_{l m}(x, y, z)=P_{l}\left(\frac{2\left(x-x_{0}\right)}{l_{x}(z)}\right) P_{m}\left(\frac{2\left(y-y_{0}\right)}{l_{y}(z)}\right),
$$

где $x_{0}, y_{0}$ - координаты центра полоски, $P_{m}-$ полиномы Лежандра.

Построены и численно реализованы БФ для неоднородностей с поперечным сечением в виде многослойных эллипсов.

$Z_{n}(z)$ - базисные функции по координате $z$. Реализовано два вида функций $Z_{n}(z)$ :

- для неоднородностей с вертикальными стенками $Z_{n}(z)=\cos \frac{(n-1) \pi z}{h}, h-$ толщина слоя с неоднородностью;

- для неоднородностей с не вертикальными стенками БФ - сплайны первого порядка $-Z_{n}(z)=\sigma_{n}^{(1)(z)}$.

Подставляем (П2) и (П3) в (П1) и проектируем на $V_{l m n}^{*}(x, y, z) ; l=0, \ldots N_{x} ; \quad m=0,2, \ldots N_{y}$; $n=0,2, \ldots N_{z}$.

В результате получим систему линейных алгебраических уравнений (СЛАУ) относительно неизвестных $X_{l m n}^{r}$.

Все интегралы в матричных элементах СЛАУ находятся в явном виде. Это позволяет перейти к третьему этапу решения. Матричные элементы СЛАУ выражаются через двойные ряды по $p, q$ (см. (П1)). Особенность ядра ОИДУ проявляется в медленной сходимости интегралов или рядов в матричных элементах полученной 
методом Галеркина СЛАУ. Основная часть компьютерного времени уходит на их суммирование. Предложен простой способ улучшения их сходимости. Суммируется заданное число членов рядов, в остальных члены рядов заменяются их асимптотикой и аналитически суммируются. Для суммирования остатка рядов используется первый член формулы Эйлера-Маклорена, т.е. суммирование по $p, q$ заменяется интегрированием по $p, q$. Такой простой способ улучшения сходимости рядов позволил сократить количество суммируемых членов ряда в 16-25 раз без потери точности. Во столько же раз сократилось время счета. Для расчетов с погрешностью (по внутренней сходимости) не более $0.1 \%$ достаточно взять 2-5 базисных функций по каждой из координат $x$ и $y$. Количество базисных функций $Z_{n}(z)$ определяется профилем и отношением общей высоты неоднородности к длине волны. Для достижения указанной точности достаточно 3-8 функций на одну длину волны вдоль неоднородности. Благодаря оптимальной организации вычислений время счета, к примеру, для бочкообразной неоднородности в 3 слоях высотой в 4 длины волны равно 2-10s при использовании процессора i3-2100 CPU 3.10GHz.

\section{2. Метод приближенных граничных (ПГУ) условий для графеновых и тонких диэлектрических, металлических, плазмонных слоев}

В этом случае поле внутри полосок не рассматривается, полоски считаются бесконечно тонкими, на них выполняются граничные условия импедансного типа. В этом приближении задача сводится к решению более простых парных интегральных или сумматорных уравнений относительно плотности тока на полоске. Сравнение результатов расчетов этими методами позволило обосновать корректность применения метода ПГУ для получения основных физических закономерностей. Обоснована корректность применения метода ПГУ для получения основных физических закономерностей и введены более точные ПГУ и понятие об эффективных размерах полосок (об эффективных размерах см. [21]).

ПГУ имеют вид

$$
H_{\mathrm{tg}}^{+}-H_{\mathrm{tg}}^{-}=\sigma / 2\left[\mathbf{n},\left(\mathbf{E}_{\mathrm{tg}}^{+}+\mathbf{E}_{\mathrm{tg}}^{-}\right)\right], \quad \mathbf{E}_{\mathrm{tg}}^{+}=\mathbf{E}_{\mathrm{tg}}^{-},
$$

где $\mathbf{n}$ - нормаль к пленке, символами „士“ обозначены компоненты поля соответственно сверху и снизу пленки, $\sigma$ - поверхностная проводимость для графена определяется формулой Кубо, для тонких диэлектрических и плазмонных слоев $\sigma=i \frac{k}{Z_{0}}(\varepsilon+1), Z_{0}-$ волновое сопротивление вакуума.

Эти приближенные граничные условия введены для неограниченных по длине и ширине графеновых слоев, они широко используются для решения задач рассеяния на ограниченных полосках.
Известными методами нетрудно получить парные сумматорные уравнения (ПСУ) относительно неизвестной плотности тока на полосках $\mathbf{j}(x, y)$

$$
\begin{aligned}
& \frac{1}{d_{x} d_{y}} \sum_{p=-\infty}^{\infty} \sum_{q=-\infty}^{\infty} \mathbf{g}_{p q} \mathbf{J}_{p q} \exp \left[i\left(\alpha_{p} x+\beta_{q} y\right)\right]+i \frac{k}{Z_{0} \sigma} \mathbf{j}(x, y) \\
& =i \frac{k}{Z_{0}} \mathbf{E}^{\mathrm{BH}}(x, y, 0), \quad x, y \in S \\
& \frac{1}{d_{x} d_{y}} \sum_{p=-\infty}^{\infty} \sum_{q=-\infty}^{\infty} \mathbf{J}_{p q} \exp \left[i\left(\alpha_{p} x+\beta_{q} y\right)\right]=0, \quad x, y \notin S ;
\end{aligned}
$$

где $S$ - поверхность, занимаемая полосками, расположенными в одной элементарной ячейке решетки, $\sigma-$ поверхностная проводимость слоя, $Z_{0}-$ волновое сопротивление,

$$
\mathbf{J}_{p q}=\int_{S} \mathbf{j}(x, y) \exp \left[-i\left(\alpha_{p} x+\beta_{q} y\right)\right] d s .
$$

Тензор $\mathbf{g}_{p q}$ определяется так же, как $\mathbf{G}_{p q}\left(z, z^{\prime}\right)$ в случае, когда точки наблюдения $z$ и $z^{\prime}$ истока расположены в одной плоскости.

Решение ПСУ ищем методом Галеркина. Рассмотрим, например, решение для полосок прямоугольной формы. Токи на полосках запишем в виде

$$
\begin{aligned}
& j_{x}(x, y)= \sum_{m=0}^{\infty} \sum_{n=0}^{\infty} X_{m n} C_{m}^{3 / 2}\left(\frac{2 x^{\prime}}{l_{x}}\right) \\
& \times\left[1-\left(\frac{2 x^{\prime}}{l_{x}}\right)^{2}\right] P_{n}\left(\frac{2 y^{\prime}}{l_{y}}\right), \\
& j_{y}(x, y)=\sum_{m=1=0}^{\infty} \sum_{n=0}^{\infty} Y_{m n} P_{m}\left(\frac{2 x^{\prime}}{l_{x}}\right) C_{n}^{3 / 2}\left(\frac{2 y^{\prime}}{l_{y}}\right) \\
& \times\left[1-\left(\frac{2 y^{\prime}}{l_{y}}\right)^{2}\right]
\end{aligned}
$$

где $x^{\prime}, y^{\prime}$ - поперечные координаты, связанные с осями полоски, $X_{m n}, Y_{m n}$ - неизвестные коэффициенты, $C_{n}^{3 / 2}-$ полиномы Гегенбауэра.

Использование метода ПГУ позволяет на порядок ускорить скорость расчетов по сравнению с методом ОИДУ. Для полосок с размерами, соизмеримыми с длиной волны в свободном пространстве, достаточно использовать 2-5 базисные функции по каждой координате. Время счета менее $1 \mathrm{~s}$.

\section{Конфликт интересов}

Авторы заявляют, что у них нет конфликта интересов. 


\section{Список литературы}

[1] D'Aloia A.G., D'Amore M., Sarto M.S. // Nanomaterials (Basel). 2020. V. 10. N 5. P. 843. doi 10.3390/nano10050843

[2] Rahmanzadeh M., Rajabalipanah H., Abdolali A. // Appl. Optics. 2018. V. 57. N 4. P. 959.

[3] Cai Y., Wang X., Guo R., Li S., Zhou Y., Xu K.-D., Joines W.T. // Nanoscale Research Lett. 2019. V. 14. N 1. P. 346.

[4] Watts C.M., Liu X., Padilla W.J. // Advanced Materials. 2012. V. 24. N 23. P. OP98. doi 10.1002/adma.201200674

[5] Barzegar-Parizi S., Ebrahimi A., Ghorbani K. // 2019. arxiv:1906.01886 [physics.app-ph].

[6] Biabanifard S., Biabanifard M., Asgari S., Asadi S., Vagoub M.C.E. // Opt. Commun. 2018. V. 427. P. 418. doi 10.1016/j.optcom.2018.07.008

[7] Xiao B., Gu M., Xiao S. // Appl. Opt. 2017. V. 56. N 19. P. 5458. doi 10.1364/AO.56.005458

[8] Huang M., Cheng Y., Chen H., Mao X., Cheng Z., Gong R. // Opt. Commun. 2018. V. 415. P. 194. doi 10.1016/j.optcom.2018.01.051

[9] Cai Y., Zhu J., Zhou J., Ye L., Liu Q.H., Lin T., Cai Z. // Optics Express. 2015. V. 23. N 25. P. 32318. doi 10.1364/OE.23.032318

[10] Alaee R., Farhat M., Rockstuhl C., Lederer F. // Optics Express. 2012. V. 20. N 27. P. 28017.

[11] Лерер А.М., Макеева Г.С. // Письма в ЖТФ. 2018. Т. 44. № 18. C. 103. doi 10.21883/PJTF.2018.18.46618.17406

[12] Лерер А.М., Макеева Г.С., Голованов О.А. // Известия вузов. Радиофизика. 2019. Т. 62. № 10. С. 787. doi 10.1007/s11141-020-10016-0

[13] Su Z., Yin J., Zhao X. // Opt. Express. 2015. V. 23. N 2. P. 1679. doi 10.1364/OE.23.001679

[14] Gao R.-M., Xu Z.-C., Ding C.-F., Yao J.-Q. // Appl. Opt. 2016. V. 55. N 8. P. 1929. doi 10.1364/AO.55.001929

[15] Li H., Wang L., Zhai X. // Sci. Rep. 2016. V. 6. P. 36651. doi 10.1038/srep36651

[16] Peng L., Li X.-M., Liu X., Jiang X., Li S.-M. // Nanoscale Advances. 2019. V. 1. N 4. P. 1452.

[17] Amin M., Farhat M., Bağci H. // Optics Express. 2013. V. 21. N 24. P. 29938. doi 10.1364/OE.21.029938

[18] Mehrabi M., Rajabalipanah H., Abdolali A., Tayarani M. // Appl. Opt. 2018. V. 57. N 14. P. 3693.

[19] Kim S., Jang M.S., Brar V.W., Mauser K.W., Kim L., Atwater H.A. // Nano Lett. 2018. V. 18. N 2. P. 971. doi 10.1021/acs.nanolett.7b04393

[20] Лерер А.М. // РЭ. 2012. Т. 57. № 11. С. 1160.

[21] Лерер А.М., Иванова И.Н. // РЭ. 2016. Т. 61. № 5. С. 435.

[22] Лерер А.М., Цветянский Е.А. // Письма в ЖТФ. 2012. T. 38. № 21. C. 77.

[23] Lerer A.M., Donets I.V., Kalinchenko G.A., Makhno P.V. // Photonics Research. 2014. V. 2. N 1. P. 31. 